\title{
Harlequin fetus - Icthyosis fetalis: Case report
}

Senai Sereke ${ }^{1}$, Semhar Berhe ${ }^{2}$, and Felix Bongomin ${ }^{3}$

${ }^{1}$ Makerere University Faculty of Medicine

${ }^{2}$ Orotta School of Medicine and Dentistry

${ }^{3}$ Gulu University Faculty of Medicine

March 30, 2021

\begin{abstract}
We are presenting a newborn with a very rare and most severe form of congenital ichthyosis that is characterized by a thick, heavily keratinized and scaly skin.
\end{abstract}

\section{Harlequin fetus - Icthyosis fetalis: Case report}

Senai Goitom Sereke ${ }^{1,2 *}$, Semhar Eyob Berhe ${ }^{1}$, Felix Bongomin ${ }^{3,4}$

${ }^{1}$ Orotta college of Medicine and health sciences, Asmara, Eritrea

${ }^{2}$ Department of Radiology and Radiotherapy, School of Medicine, Makerere University College of Health Sciences, Kampala, Uganda

${ }^{3}$ Department of Medicine, School of Medicine, Makerere University College of Health Sciences, Kampala, Uganda

${ }^{4}$ Department of Medical Microbiology and immunology, Faculty of Medicine, Gulu University, Gulu, Uganda

*Corresponding author

Email Addresses:

Senai Goitom Sereke: nayhersen@gmail.com

Semhar Eyob Berhe: semhareyobmd@gmail.com

Felix Bongomin: drbongomin@gmail.com

\section{Corresponding author address:}

Department of Radiology and radiotherapy, Makerere University college of Health sciences

Kampala Uganda; Mobile: +256-786-271-349

\section{Abstract}

A newborn born with a very rare and most severe form of congenital ichthyosis that is characterized by a thick, heavily keratinized and scaly skin.

Clinical message

Icthyosis fetalis is a very rare form of skin disorder that is very difficult to treat, especially in low resource settings. 


\section{Case Presentation}

Harlequin fetus or Harlequin is a very rare and most severe form of congenital ichthyosis characterized by a thick, heavily keratinized and scaly skin $(1,2)$.

A $3.3 \mathrm{~kg}$ term male neonate with Apgar scores of 8 and 9 at one and 5 minutes respectively was delivered to a 25-year-old Eritrean woman by caesarean section. The baby was covered with thick yellowish to whitish scales, split by extensive some deep and others shallow fissures extending to the dermis. The scale covered almost the entire body. There was severe ectropion. There was scalp hair, the limbs were edematous and inflexible digits due to taut skins (Figure 1A, B and C). The pregnancy was uneventful. Both parents were paternal first-degree cousins. The baby was admitted to neonatal intensive care. In the first day, the baby was suckling well and was well hydrated. On the second day, the scales started to change in color (Figure 1D) and neonatal sepsis ensued. He was intravenous antibiotic, but he progressively deteriorated. The parents demanded, they wanted to take their baby home and they were discharged eventually against medical advice.

\section{References}

1. Hovnanian A. Harlequin ichthyosis unmasked: a defect of lipid transport. J Clin Invest. 2005 Jul;115(7):1708-10.

2. Liang Q, Xiong F, Liang X, Zheng D, Su S, Wen Y, et al. Two successive cases of fetal harlequin ichthyosis: A case report. Exp Ther Med. 2019 Jan;17(1):449-52.

\section{Figure legend}

Figure A, B and C (day zero) and D (day one). A, B, C demonstrated yellowish to whitish scales split by extensive deep and shallow fissures extending to the dermis. Widely open mouth, severe ectropion, edematous limb with inflexible digits due to taut skins was also demonstrated. D demonstrated that decrement of the yellowish scale with more exposure of the fissures and drying of the fissures edge.

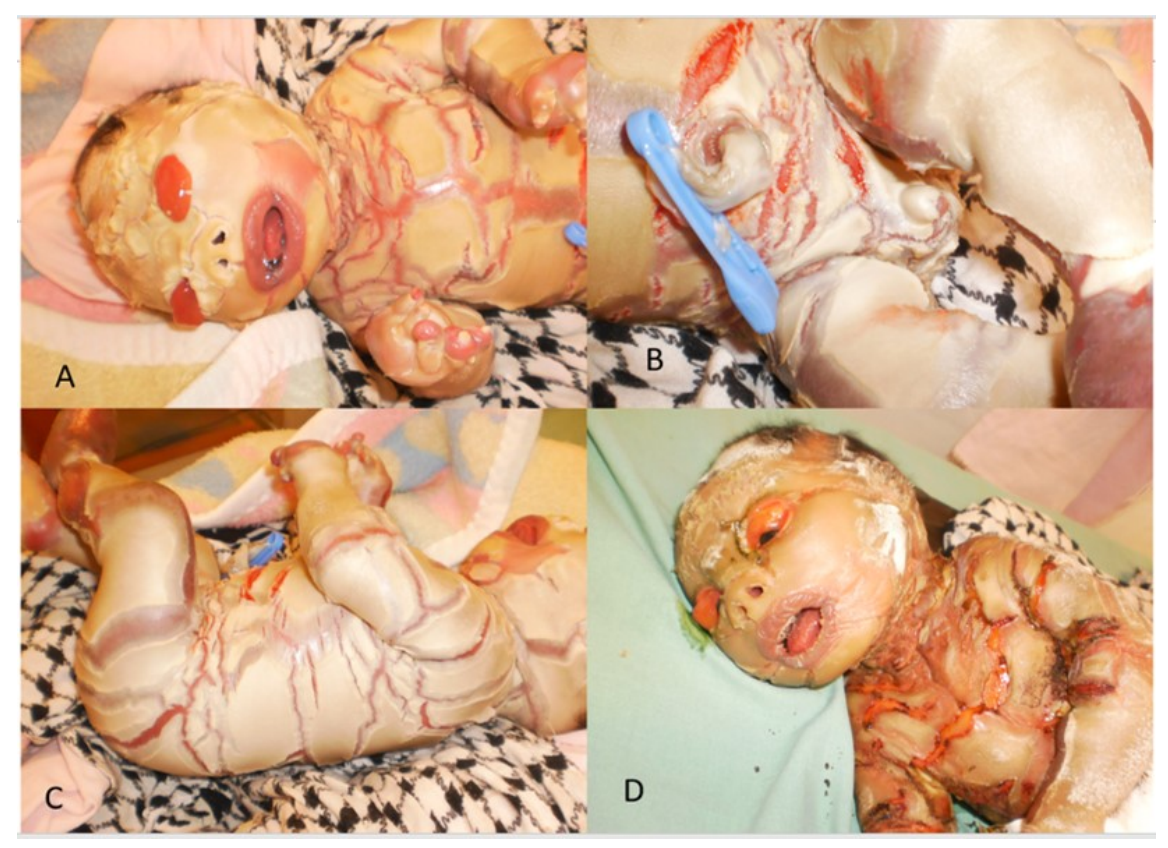

